\title{
Effects of infrasound generated in urban areas on health of people and animals - an attempt to localize environmental infrasound sources using computer simulations
}

\author{
Grzegorz Nowicki', Tomasz Jerzy Nowicki ${ }^{2}$, Andrzej Prystupa ${ }^{3}$, Barbara Ślusarska ${ }^{4}$, Ewa Chemperek ${ }^{1}$ \\ ${ }^{1}$ Department of Expert Medical Assistance with Emergency Medicine Unit, Medical University of Lublin, Poland \\ ${ }^{2}$ Department of Structural Mechanics, Faculty of Civil Engineering and Architecture, Lublin University of Technology of \\ Lublin, Poland \\ ${ }^{3}$ Department of Internal Medicine, Medical University of Lublin, Poland \\ ${ }^{4}$ Chair of Development in Nursing, Faculty of Nursing and Health Sciences, Medical University, Lublin, Poland
}

Nowicki G, Nowicki TJ, Prystupa A, Ślusarska B, Chemperek E. Effects of infrasound generated in urban areas on health of people and animals - an attempt to localize environmental infrasound sources using computer simulations. J Pre-Clin Clin Res. $2014 ; 8(2)$ : 81-85. doi: $10.5604 / 18982395.1135655$

\begin{abstract}
Introduction. Inaudible but ubiquitous environmental infrasound undoubtedly affects the frame of mind and health of humans.

Objective. The object of the study is to prove that civil engineering objects can be sources of infrasound, and a review of the literature on effects of infrasound on the health of people and animals.

Methods. Computer simulation of an air flow around a typical element of outdoor infrastructure. The simulation was carried out using the author's computer programme, based on Discrete Vortex Method algorithms.

Results and conclusions. The numerical experiment clearly proved that industrial objects can be sources of infrasonic noise during windy weather. The picture of air flow around a cylinder shows that regular turbulent motion is a source of sound with one dominating frequency. The sound once generated propagate far from its source. Frequency and intensity of the sound depends on wind velocity and shape of the object. Further research in this area are advisable.
\end{abstract}

\section{Key words}

infrasound, health effects, computer simulations

\section{INTRODUCTION}

The human ear has the ability to perceive sound frequency ranges from $20-20,000 \mathrm{~Hz}[1,2]$. The borders may vary due to personal predisposition, state of health and environmental conditions. Sound below $20 \mathrm{~Hz}$ is called infrasound and its effects on living organisms have been studied for the last decades. Nowadays, it is commonly accepted that people and animals exposed to infrasound can develop health problems.

Although infrasound noise is usually connected with industrial expansion, it is also a natural phenomenon. Natural sources of infrasound may be both of an inanimate and animate nature. Sound within a wide range of frequency, including these under $20 \mathrm{~Hz}$, always accompany widely understood tectonic activity. Constantly dripping water (including ice) or a wavy surface of water usually spreads a broad spectrum of acoustic effects exceeding the limits of audible sounds. Meteorological phenomena, such as wind, rain or atmospheric discharge, take place in atmospheric air so that they cannot occur without noise, including its infrasonic part.

The second group of infrasonic sources are animals. Big animals, such as elephants or whales, are known to

Address for correspondence: Grzegorz Nowicki, Department of Expert Medical Assistance with Emergency Medicine Unit, Medical University of Lublin, ul. S. Staszica 6, 20-081 Lublin, Poland

E-mail: grzesiek_nowicki@interia.pl

Received: 05 August 2014; accepted: 03 November 2014 communicate using low frequencies of sound. The regular walking of an animal or a human also generates infrasound. All of the enumerated sources of infrasound are natural and it is almost certain that they are not harmful, and it is industrial growth which has caused increased interest in infrasound. There is no doubt that machinery (engines, vehicles, etc.) is the source of the most dangerous infrasound. Much research has been carried out in order to determine the infrasonic environment in the vicinity of machines (including interiors of vehicles), and to discover its influence on people. The knowledge in this field seems to be extensive [3].

Although infrasound has been studied for a long time, there is still one group of its sources that seem to be overlooked the group constituted by the urban environment. Buildings such as skyscrapers or towers, outdoor industrial installations (e.g. pipelines, chimneys) or transport infrastructure (bridges, long free-standing walls, e.g. noise barriers). All such objects as sources of aerodynamic turbulence emit sound during windy weather. The frequency and intensity of the sound depends on shape of the object, its neighbourhood and wind speed. Computer simulation has showed that even at moderate wind speed infrasound generation is possible for a typical element of urban environment. This group of infrasonic noise sources can be placed between the two previous groups, because sound is generated by wind but the objects are artificial and not occur in nature. This study investigates environmental sources of infrasound and its influences on people and animals. 


\section{OBJECTIVE}

The object of the study, based on numerical analysis and computer simulation on a simplified model, to prove that civil engineering objects can be sources of infrasound.

\section{MATERIALS AND METHOD}

The object of the study is sound generated aerodynamically, and focuses on noise generated as a by-product of an airflow. Due to high costs of field measurements, computer simulations have been chosen for preliminary research.

Airflow, as an example of fluid flow, is governed by a Navier-Stokes equation, which is a differential equation that still challenges scientists and theoretical engineers. Exact analytical solutions of the equation are known for simple cases only, and is the reason why numerical computer simulations are commonly used to explore fluid flows and their effects. The most commonly used methods here are the Finite Volume Method and Finite Element Methods, called classical methods nowadays. Due to the fact that the precision of sound emission estimation depends crucially on the accuracy of the vorticity field reconstructed on the grounds of fluid motion velocity field, the two classical numerical meshbased methods used for solving the Navier-Stokes equation usually fail to show which flows can generate sounds. This is a problem often neglected in engineering simulations. Audible sounds are usually explored using different methods, but inaudible sounds are seldom taken into consideration. The Discrete Vortex Method (DVM) is an alternative to the classical methods, in which the finite element mesh is not used, and it is not the velocity but vorticity field that is simulated directly, which suits the method well for sound emission estimation. In addition to the quantitative output, each simulation of fluid flow results in a good visualisation of turbulence given in a time scale. The visualisation allows assessment of the sound emission (including inaudible sounds) taking place. The DVM method, however, is less popular than the classical methods. Currently, there is no computer software available (commercial or open source) for performing computer simulations of fluid flow using the method, and all implementations of DVM still remain at the stage of academic research. One such implementation is the computer program 'Ventus' developed in the Department of Structural Mechanics at University of Technology in Lublin, Poland [4]. The programme was the research tool used in the presented study.

The Discrete Vortex Method (DVM) is a numerical method developed for solving the Navier-Stokes equation, based on the Lagrangian model of a particle tracing $[4,5]$. In DVM, the equation is solved by direct computer simulation of a physical phenomena. A finite mesh, known from the finite element and finite volume methods, is not applied in DVM.

Considering 2D areas of fluid flow and assuming a homogeneous dry air with a constant density, the following form of the N-S (Navier Stokes) equation can be used to describe the phenomenon of interest:

$$
\frac{\partial \mathbf{u}}{\partial t}+(\mathbf{u} \cdot \nabla) \mathbf{u}=-\frac{1}{\rho} \nabla p+v \Delta \mathbf{u},
$$

where: $\mathrm{u}$-velocity field, $\mathrm{p}$-pressure field, $\rho$-density, t-time $v$-kinematic viscosity. The part $(\mathbf{u} \cdot \nabla)$ is the operator.
The equation (1) is decomposed by calculation of the rotation of the vector $\mathrm{u}$, which gives the so-called vorticity transport equation:

$$
\frac{\partial \boldsymbol{\omega}}{\partial t}+(\mathbf{u} \cdot \nabla) \boldsymbol{\omega}=v \Delta \boldsymbol{\omega}
$$

where $\boldsymbol{\omega}$ is a vorticity field of the flow. The last equation (2) is composed of two components: advection (3) and diffusion (4) one:

$$
\begin{gathered}
\frac{\partial \boldsymbol{\omega}}{\partial t}+(\mathbf{u} \cdot \nabla) \boldsymbol{\omega}=0, \\
\frac{\partial \boldsymbol{\omega}}{\partial t}=v \Delta \boldsymbol{\omega} .
\end{gathered}
$$

The separation let us treat fluid flow as two simultaneous and independent phenomena: advection and diffusion, and is known as Split Algorithm. In DVM it is not the velocity field which is being directly simulated, but its vorticity constituent. The vorticity field is represented in a discrete form, which for the $2 \mathrm{D}$ case reduces to a set of particles, a so-called vortex cloud. Each vortex particle contribute to then total cloud velocity field of the flow, that it co-represents, under the aerodynamic BioSavart law. Assembly of the cloud velocity field and potential velocity field not included in equation (2) but present in equation (1) gives the final velocity field in which the vortex cloud is drifted. The process is described by the advection formula (3), while the diffusion (4) is implemented as the vortex cloud erosion caused by viscosity of the fluid medium.

The source of vorticity in the flow is a solid body immersed in it, precisely the solid-fluid border. Because fluid velocity at the border is zero, there is a need to cover the body with a vortex sheet that reduces the influence of the environment outside the body. The sheet is created and shed at every simulation step. Vortex particles that have been shed become members of the vortex cloud. To sum up it, must be stated that DVM is well suited to the computer modelling of turbulent flows.

\section{RESULTS}

The computer simulation was performed using Discrete Vortex Method (DVM), a numerical method developed for solving the Navier-Stokes equation [5], which describes the motion of fluid continues substance. Solutions of the equation usually give fields of velocity and pressure of unsteady fluid medium. The output can be used subsequently for estimating sound emission $[6,7]$.

The case taken into consideration concerns the wind flow round a long straight cylinder (Fig. 1) of $0.2 \mathrm{~m}$ in diameter under a wind attack of $5 \mathrm{~m} / \mathrm{s}(18 \mathrm{~km} / \mathrm{h})$, such as a long cable or a thin pipeline. Model tests are well established methods of wind engineering. They allow the obtaining of reliable results by means of wind tunnel laboratory tests or numerical simulations $[8,9]$. The long tradition of model research in wind science has arisen due to the high cost of field study. The computer simulation included mutual interaction between the solid body and fluid. The air stream was assumed to be non-turbulent before encountering the cylinder, so only the vorticity that appeared in the flow came from the presence of the solid body in the stream. Figure 2 presents a fully developed vortex street on the leeward side of the cylinder. The street vortex is a well known form of turbulence called 


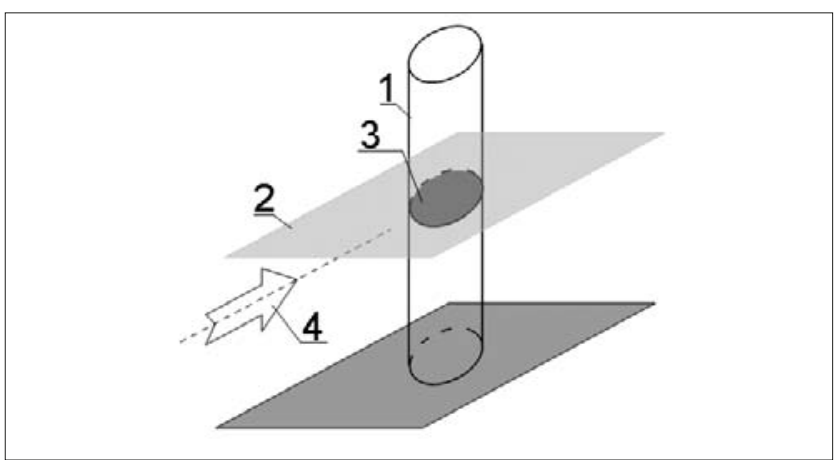

Figure 1. View of the object under aerodynamic analysis: 1 - long cylinder, 2 - DVM analysis plane, 3 - section from Fig. 3, 4 - wind attack

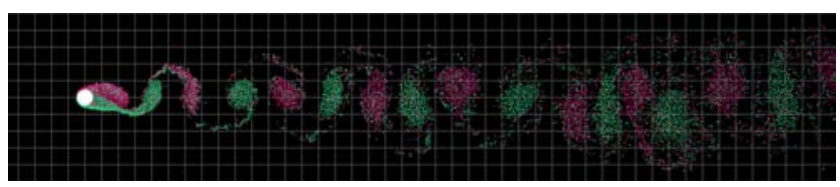

Figure 2. Computer simulation of wind flow over a long cylinder (Source: own research)

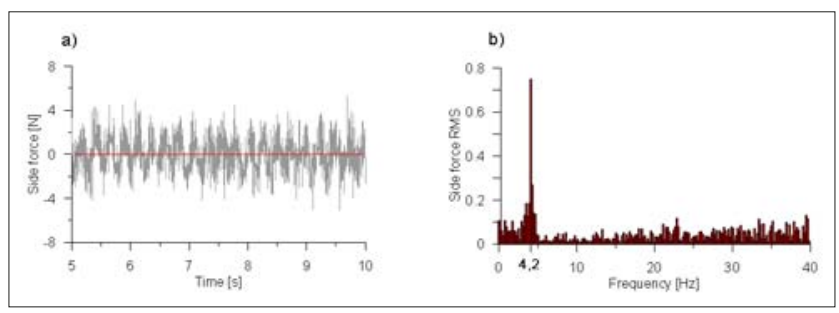

Figure 3. Side forces affected by the cylinder from Fig. 2: a) time history of the force b) amplitude spectrum

the von Karman vortex street. The colour indicates the turn of vorticity; red has been used for clockwise vorticity, green for counterclockwise. The phenomena consists in shedding vortices in a regular way. The next vortex always has inverse spin to the previous one. Each vortex that appear in the stream causes decrease or increase in air pressure. Pressure jumps occur close to the solid body, which makes it a source of sound. The sound then propagate far its source $[6,7]$. If the frequency of vortex shedding drops below $20 \mathrm{~Hz}$, then the sound is called infrasound and is usually referred to as being below the threshold of human hearing.

Vortex shedding is not the only source of sound but also induces side aerodynamic force, which is easily calculated by the 'Ventus' programme. Figure 3a shows how the force changes in time, while figure $3 \mathrm{~b}$ is an amplitude spectrum of the signal (Fig. 3a). The dominating frequency is $4.2 \mathrm{~Hz}$. Because the same vortexes generate sound, the sound is of the same frequency $-4.2 \mathrm{~Hz}$, which means that it is infrasound generated under the level of human perception.

The case of the cylinder that has been analyzed may be referred to real civil engineering structures. Practically, each long element of the building structure with a circular crosssection and diameter of $0.2 \mathrm{~m}$ (or close to $0.2 \mathrm{~m}$ ) placed in free space generates a vortex street, which results in infrasound emission during wind at speed $5 \mathrm{~m} / \mathrm{s}$ (or similar). Such an element can be one of cables used in cable-stayed or suspended structures: bridges, footbridges, large-surface roofs. Similar cables are parts of high masts and the base structure of a cable railway. The same effect is created by a pipeline suspended on pylons. All the building are common in urban areas, but probably do not complete urban environmental infrasound sources. Although it goes beyond the presented research, it is high probable that long obstacles in a flat terrain, such as a regular drop (e.g. cliff, earth embankment) or long free-standing wall (e.g. baffle board) are another group of infrasound sources because they too are able to generate vortex speed as specific wind speeds.

\section{DISCUSSION}

Infrasound is not audible for humans, based on its definition, but it is accepted to be sensed by the body. Infrasound can result in human body functional disturbance, even injury [10]. Industrial wind turbines can harm human health if sited close to residents. Harm can be avoided if wind turbines are situated at an appropriate distance from humans. The infrasound effects studied were on the cardiovascular (myocardium) and nervous systems, eye structure, hearing and vestibular function, and endocrine modulation. Specific central nervous system (CNS) effects studied included annoyance, sleep and wakefulness, perception, evoked potentials, electroencephalographic changes, and cognition.

Volunteer studies investigating the potential effects of infrasound are relatively limited, and the quality of many of them is questionable, and they yield conflicting results. Very few studies appear to have been published in recent years. Most of the studies identified involved infrasound exposures at $90 \mathrm{~dB}$ and higher, and ranged from minutes to several months. Of the many animal studies identified, there were none involving long-term (six months or more) exposure. There were few studies evaluating reproductive function, developmental effects, and immunological effects, and no studies that evaluated carcinogenic effects. The primary effect of infrasound in humans appears to be annoyance. Aural pain and damage (i.e., scarring of the tympanic membrane and vascular infection of the eardrum membrane) have been reported [11].

Infrasound (i.e. $<20 \mathrm{~Hz}$ for humans) is not audible, but exposure to high-levels of infrasound will produce large movements of cochlear fluids. Infrasound entering the ear through the ossicular chain is likely to have a greater effect on the structures of the inner ear than sound generated internally. Exposure to infrasound of about $90 \mathrm{~dB}-\mathrm{G}$ can cause excessive fatigue, drowsiness and noise-induced hearing loss [12]. In animals exposed to $4 \mathrm{kHz}$ OBN (octave band of noise) at $108 \mathrm{~dB}$ SPL, there were larger functional losses and much greater hair-cell loss in the cochlea. The presence of infrasound during this intense $4 \mathrm{kHz}$ OBN exposure increased cochlear damage because infrasound caused more intermixing of cochlear fluids through the damaged reticular lamina. Simultaneous infrasound and a moderate $4 \mathrm{kHz}$ OBN did not increase cochlear damage because the reticular lamina rarely breaks down during this moderate level of exposure [13].

Studies of 1,063 residents in multifamily buildings in Sofia, Bulgaria, experiencing a noise level above $60 \mathrm{dBA}$ and infrasound levels from 55 to $78 \mathrm{~dB}$, found a statistically significantly increased percentage of persons with psychosomatic complaints (e.g., weakness and fatigue) and sleep disturbance (e.g., restlessness during sleep) versus those exposed to a lower level noise and infrasound [14]. 
Rats exposed to infrasound for 60 days showed biochemical and morphological changes in blood and tissues, including dystrophic tissue changes in the lungs, liver, kidneys, heart, adrenals, and testicles [15].

Some people with wind turbines located close to their home have reported a variety of clinical symptoms that in rare cases are severe. These symptoms include sleep disturbance, headaches, difficulty in concentrating, irritability and fatigue, as well as a number of otologic symptoms, including dizziness or vertigo, tinnitus and the sensation of aural pain or pressure. The symptom group has been termed 'wind turbine syndrome' and speculated to result from the low frequency generated by wind turbines [16].

Pierpont documented symptoms reported by individuals exposed to wind turbines, which include sleep disturbance, headache, tinnitus, ear pressure, dizziness, vertigo, nausea, visual blurring, tachycardia, irritability, problems with concentration and memory, and panic episodes associated with sensations of internal pulsation or quivering when awake or asleep.

Nissenbaum et al. used validated questionnaires in a controlled study of two marine wind energy projects. They concluded that the noise emissions of wind turbines disturbed sleep, caused daytime sleepiness, and impaired mental health in residents living within $1.4 \mathrm{~km}$ of the two wind turbines installation under study [17].

Several studies have investigated the possible effects of infrasound on the cardiovascular system. Borredon and Nathie reported elevation in diastolic blood pressure in a male exposed to infrasound for $50 \mathrm{~min}$ [18]. Standberg et al. found exposure to infrasound for one hour increased diastolic blood pressure, decreased systolic blood pressure and increased pulse rate [19]. Another study reported that exposure to infrasound reduced the strength of cardiac contraction. In addition, behavioural and neurophysiological changes were observed.

Rats and guinea pigs (5 test animals, 2 controls per group) were exposed to infrasound for $3 \mathrm{~h} /$ day for 45 days; and tissues were collected on days $5,10,15,25$, and 45 for pathomorphological examination. A single exposure of $4-10 \mathrm{~Hz}$ at $120-125 \mathrm{~dB}$ led to short-term arterial constriction and capillary dilatation in the myocardium. Prolonged exposure led to nuclear deformation, mitochondrial damage [20].

Twenty healthy males were exposed to infrasound for $20 \mathrm{~min}$. in one series of experiments and then for one hour on alternate days in a second series of experiments. Infrasound was observed to increase diastolic blood pressure, with the most significant effect seen with $16 \mathrm{~Hz}$, and a maximum mean increase of $8 \mathrm{~mm} \mathrm{Hg}$ occurred after 30 minutes, and a decrease in systolic blood pressure and pulse rate. This suggests that peripheral vasoconstriction with increased blood pressure was induced with acute infrasound stimulation [21].

Researches have shown that infrasound exposure causes functional and structural disorders of the central nervous system, which lead to lower work efficiency, poor memory and easily provoked agitation.

Brain injury observed in rats exposed to infrasound at high sound pressures may be due to elevated glutamate concentrations and its exciting toxicity. Four groups of rats were exposed to infrasound. Significant increases in brain glutamate were observed in rats exposed at $120 \mathrm{~dB}$, with the effect being more pronounced in the rats exposed to $16 \mathrm{~Hz}[22]$.
Infrasound is not audible, but exposure to a high-level of infrasound will produce large movements of cochlear fluids. Lim et al. were the first to examine in detail the histopathology of the middle and inner ears following exposure to infrasound. They found a number of pathological changes including rupture of the tympanic membrane and middle-ear bleeding [23]. Hensel et al investigated the impact of infrasound on cochlea function by measuring distortion product otoacoustic emissions. The cochlea is the auditory portion of the inner ear. Infrasound induced changes in sound processing by the cochlea, but did not cause damage to the outer hair cells [24].

Landström et al. investigated the effects of $15 \mathrm{~min}$. infrasound at EEG frequencies on wakefulness in six volunteers. Changes in EEG were variable and none was significant, although there was a suggestion that theta activity increased with the eyes closed [25]. When 18 healthy male students were exposed to infrasound during sleep, the pattern of sleep was little affected. In contrast, audible sound (i.e. synthesized traffic noise) had more 'harmful effects' on sleep (e.g. caused difficulty in initiating sleep and affected reaction rates after exposure) [26].

Infrasound induced adverse bioeffects on reproduction depend on the exposure parameters. Serum testosterone concentration of rats exposed to infrasound for one day decreased significantly compared to control. Exposed to infrasound, rats of all experimental groups displayed significantly increased latency to first mount or ejaculation, and decreased the numbers of mount or ejaculation compared to control, and with prolonged exposure, sexual behavior was reduced more profoundly [27].

\section{CONCLUSIONS}

The presented numerical experiment proved clearly that industrial objects can be sources of infrasonic noise during windy weather. The picture of air flow around a cylinder shows that regular turbulent motion is a source of sound with one dominating frequency. The sound once generated propagated far from its source. Frequency and intensity of the sound depends on the velocity of wind and shape of the object. Further researche in this area are advisable.

\section{REFERENCES}

1. Tuleuhanov ST, Desouky OS, Mohaseb AM. The influence of the infrasound on the immunological properties of rats blood. J Biophys. 2010; 20(3): 245-255.

2. Kurpas D, Mroczek B, Karakiewicz B, Kassolik K, Andrzejewski W. Health impact of wind farms. Ann Agric Environ Med. 2013; 20(3): 595-604.

3. Nowicki G, Mitraszewska I, Kamiński K, Wierzejski A. Research of Infrasound Noise in Heavy Goods Vehicle and Busses. In: Kabashkin IV, Yatskiv IV (eds.). Proceedings of the 8th International Conference Reliability and Statistics in Transportation and Communication (RelStat'08); Oct 15-18 2008; Riga.

4. Nowicki T. Influence of Boundary Condition Implementation in Discrete Vortex Method on Aeroelastic Response of Bridge Decks. Monographs - Lublin University of Technology, 2012.

5. Lewis RI. Vortex Element Methods for Fluid Dynamics Analysis of Engineering Systems, Cambridge University Press, 2005.

6. Lighthill MJ. On sounds generated aerodynamically, Part I: General theory. Proc Roy Soc. London 1952; A241: 564-87.

7. Lighthill MJ. On sounds generated aerodynamically, Part II: Turbulence as a source of sound. Proc Roy Soc. London 1954; A222: 1-32. 
8.Lipecki T, Flaga A. Vortex excitation model. Part I. mathematical description and numerical implementation. Wind and Structures 2013; 16(5): 457-476

9. Lipecki T, Flaga A. Vortex excitation model. Part II. application to real structures and validation. Wind and Structures 2013; 16(5): 477-490.

10. Landstrom U. Laboratory and field studies on infrasound and its effects on human. J. Low. Freq. Noise. Vib. 1987; 6(1): 29-33.

11. Broner N. The effects of low frequency noise on people - A review. J Sound Vib. 1978; 58(4): 483-500.

12. Futatsuka M, Maeda S, Inaoka T, Nagano M, Shono M, Mi-yakita T. Whole-body vibration and health effects in the agricultural machinery drivers. Ind. Health. 1998; 36:127-32.

13. Harding GW, Bohne BA, Lee SC, Salt AN. Effect of infrasound on cochlear damage from to a $4 \mathrm{kHz}$ octave band of noise. Hear Res. 2007; 225(1-2): 128-138.

14. Radneva R. Studying the effect of acoustic conditions in the living environment of multifamily buildings on inhabitants. Khig Zdraveopazvane. 1997; 40 (3-4): 40-44.

15. Dadali VA, Svidovyi VI, Makarov VG, Gorkova LB, Kuleva VA, Pavlova RN, Tarasova OV, Timofeeva VM. Effects of infrasound and protective effect of adaptogens in experimental animals. Gig Sanit. 1992; (1): 40-43.

16. Pierpont N. Wind turbine syndrome. K-selected books http://www. kselected.com/ (access: 2013.09.29).

17. Nissenbaum MA, Aramini JJ, Hanning CD. Effects of industrial wind turbine noise on sleep and health. Noise Health 2012; 14(60): 237-243.
18. Borredon P, Nathie J. Physiological reactions of human subjects expose to infrasounds. Rev Med Aeronaut Spat. 1973; 12(46): 276-279.

19. Standberg UD, Bjerle P, Danielsson A, Hornqwist-Bylund S, Landström U. Studies of circulation changes during exposure to infrasound. Arbetarskyddsstyrelsen, Publikationsservice, Solna, Sweden 1986: 29.

20. Alekseev SV, Glinchikov VV, Usenko VR. Myocardial ischemia in rats exposed to infrasound. Gig Tr Prof Zabol. 1983; (8): 34-38.

21. Danielsson A, Landstrom U. Blood pressure changes in man during infrasonic exposure. An experimental study. Acta Med Scand. 1985; 217(5): 531-535.

22. Guo-You F, Jing-Zao C, Ke-Yong J. Changes of glutamate in brain of rats exposed to infrasound. J Fourth Mil Med Univ. 1999; 20(4): 288-290.

23. Lim DJ, Dunn DE, Johnson DL, Moore TJ. Trauma of the ear from infrasound. Acta. Otolaryngol. 1982; 94(3-4): 213-231.

24. Hensel J, Scholz G, Hurttig_U, Mrowinski D, Janssen T. Impact of infrasound on human cochlea. Hear Res. 2007; 233(1-2): 67-76.

25. Landström U, Byström M, Byström N. Changes in wakefulness during exposure to noise at $42 \mathrm{~Hz}, 1000 \mathrm{~Hz}$ and individual EEG frequencies. Jnl Low Freq Noise Vibn. 1985; 4: 27-33.

26. Okada A, Inaba R. Comparative study of the effects of infrasound and low frequency sound with those of audible sound on sleep. Environ Int. 1990; 16(4-6): 483-490.

27.Zhuang Z, Pei Z, Chen J. Infrasound-induced changes on sexual behavior in male rats and some underlying mechanisms. Environ Toxicol Pharmacol. 2007; 23(1): 111-114. 